\title{
Repercusiones de \\ la teoría de Piaget \\ en los diseños curriculares
}

\section{Francisco Marro *}

Los intentos de recoger los avances derivados de la investigación científica en la programación educativa no se corresponden siempre con los objetivos de los científicos al emprender su investigación. En este artículo se analizan las repercusiones de Piaget en los diseños curriculares de nuestro país y se valora críticamente los errores en que se incurre al extrapolar sin más esa teoría al contexto educativo.

" Dirección del autor: Paseo San Juan, 38, principal. Barcelona-10 


\section{Introducción}

Las referencias a la obra de Piaget en nuestros ambientes educativos no cesan de aumentar. Sin embargo, esta utilización de la teoría genética no está fundamentada la mayoría de las veces en una doble reflexión acerca de la naturaleza de la educación y dé la verdadera significación de las aportaciones psicológicas de Piaget.

Decididos a abordar el estudio de la aplicabilidad y las repercu. siones de la psicología genética en la educación, vimos, en conse. cuencia, conveniente ahondar en la naturaleza de la educación, y ello nos obligó:

- en primer lugar, a profundizar en las diferentes posturas epis. temológicas que intentan precisar la estructura formal de las Ciencias de la Educación (Ciencias de la Educación concebidas como un campo práctico de saber, ciencia específica de la Educación concebida como una Praxiología y ciencia especifica de la Educación construída a través de la interdesciplina. riedad) ${ }^{1}$

- en segundo lugar, a reflexionar sobre el estatuto de la Psicología de la Educación en el marco de las Ciencias de la Educa. ción

- y, en tercer lugar, a optar por una de las tres posturas (extrapolación-traducción, independencia, interdependencia-interacción) posibles acerca de cómo construir el saber específico de la Psicología de la Educación. (Coll, C. 1980).

También llevamos a cabo paralelamente un estudio acerca de la verdadera significación de la psicología genética dentro de todo el marco epistemológico en el que se inserta.

En este artículo vamos a limitarnos a exponer una parte de los resultados obtenidos a nivel de repercusiones de la obra de Piaget en nuestro ambiente educativo. El objeto de este apartado era describir y analizar las distintas representaciones existentes de las aportaciones piagetianas y su utilización real. Más concretamente, se trataba de responder a estas preguntas:

- iHa habido una verdadera influencia de la obra de Piaget en nuestro ambiente educativo?

- En caso afirmativo, cqué sectores de la enseñanza han sido los más permieables a las ideas piagetianas? y ccuáles son las principales dimensiones de tal influencia?

- ¿Cuál es la pertinencia de estas innovaciones educativas con respecto a la teoría de origen? 
Con objeto de delimitar nuestro campo de trabajo, redujimos nuestro análisis a las influencias en los niveles de Preescolar y Ciclo Inicial. Las dos suposiciones básicas al iniciar nuestro trabajo eran, en primer lugar, que los aspectos más conocidos de la teoria genéti. ca serían los estructurales, y, en segundo lugar, que hallariamos una tendencia clara hacia una concepción extrapolativa de las ideas pia. getianas a la educación.

Cuando hablamos de aspectos estructurales hacemos referencia al interés epistemológico que presidió las investigaciones de la Escuela de Ginebra hasta fines de la década del 60. Interesado por resolver el problema de la relación de disyunción o irreductibilidad y, al mis. mo tiempo, adecuación o coordinación existente entre el conocimiento lógico-matemático y el conocimiento empírico, Piaget se propone abordar la cuestión mediante una metodología genética, es decir, estudiando la génesis de los conocimientos científicos. La pre. gunta que se plantea es: $¿$ De qué manera el pensamiento científico pasa de un estado de menor conocimiento a un estado de conocimiento reconocido como superior por la comunidad científica en un momento histórico determinado? Así, de lo que se tratará será de estudiar de manera inductiva, a título de hechos que se suman a otros hechos, el acrecentamiento de los diferentes tipos de conoci. miento científico, para poder llegar a elaborar una teoría de los mecanismos comunes, de las convergencias de estos diversos acrecen. tamientos. El objetivo de la epistemología genética será, pues, estu. diar cómo se construyen las normas, los sistemas normativos del hombre adulto, para deducir su validez y significado para el campo científico considerado.

En primer lugar, Piaget estudia la evolución de las nociones de un determinado campo científico reconstruyéndolas históricamente (método histórico-crítico). Este método no le permite llegar a las for. mas elementales o intuitivas de las nociones porque la evolución de una ciencia es obra de mentes adultas. Para resolver este problema adopta el método psicogenético, estudiando el niño para compren. der mejor al adulto. De esta forma, la psicología genética asume el papel de describir cómo se van construyendo los límites «a priori), las categorías intelectuales del conocimiento en el curso de la evolución intelectual del individuo entre el nacimiento y la edad adulta. El análisis histórico-crítico facilita el estado actual de desarrollo de una noción determinada en el pensamiento científico adulto. Este estado actual de la noción constituye el marco de referencia en el que se inserta el análisis psicogenético. Este marco es el que determina los problemas específicos que serán abordados a nivel psicológico. La lógica es el tercer instrumento metodológico de Piaget. La formali. 
zación lógica asume el papel de fundamentar la validez de los sistemas normativos que ha hecho patentes el análisis psicogenético.

Piaget llega a demostrar que las estructuras espontáneas que caracterizan el período de las operaciones formales, coinciden con las estructuras que están en la base de la reconstrucción boubakista de la totalidad de las matemáticas. Con esta demostración logra su objetivo de explicar la adaptación de los modelos matemáticos a la realidad física al (c...restablecer la continuidad entre las reorganizaciones inconscientes de las etapas iniciales de la inteligencia, y las construcciones explícitas y pensadas del pensamiento adulto). (Ce llerier, 1978, p. 33.)

Debido a este marco epistemológico en el que se insertan, las investigaciones psicológicas piagetianas no estudian las conductas de los individuos en sí mismas, sino según un modelo de estadios. No se estudian los aspectos funcionales de las operaciones. Sólo interesan, las anticipaciones, las inferencias verbales, que el sujeto hace ante una situación determinada, y no tanto sus realizaciones efectivas (éstas sólo tenian la misión de confirmar o refutar las hipótesis que el experimentador se hacía respecto a las primeras). En definitiva, pues, se intentaba describir lo más general, lo común a las estruc. turas intelectuales de los sujetos de un mismo nivel de desarrollo. Esta centración en lo común hizo que se descuidasen en estas investigaciones la variabilidad interindividual originada por factores de tipo diferencial o ambiental.

Con el término extrapolación aludimos al significado que Coll da a este término:

\footnotetext{
«La versión más radical de esta hipótesis reposa sobre la idea de que los resul. tados de las investigaciones realizadas en una perspectiva estrictamente psico. lógica puede ser extrapolados a la situación educativa; es decir, se parte del principio que afirma que las relaciones entre variables establecidas o postuladas por los estudios psicológicos pueden servir de base para predecir la existencia del mismo tipo de relaciones entre dichas variables cuando se presentan en el contexto educativo). (Coll, C. 1980, p. 44).
}

Las reflexiones educativas hechas sobre la base de esta concepción tenderá a llevar a cabo-una recolección de las aportaciones piagetianas y a transplantarlas a una realidad que es muy diferente a la que está en su origen sin someterlas a ninguna modificación.

\section{METODO}

Con el fin de recoger la información pertinente para responder a los interrogantes planteados, elaboramos un plan de trabajo que contenía los siguientes apartados: 
a) Análisis de la documentación oficial, a nivel de Cataluña, que recogiese orientaciones didácticas y programaciones destinadas a alumnos de Parvulario y Ciclo Inicial.

b) Entrevistas destinadas a recoger opiniones de profesionales de diferentes sectores de la enseñanza: política educativa, formación del profesorado de E.G.B.; reciclaje y práctica educativa.

c) Pase de un cuestionario dirigido a una muestra representati. va de centros públicos barceloneses y, más concretamente, a todos los profesores de Parvulario y Ciclo. Inicial de dichos centros. -

En este artículo nos limitaremos a exponer los resultados del primero de estos apartados, es decir, el referente al análisis de la documentación oficial que ya ha sido publicada ${ }^{2}$. El texto seleccionado para este fin es el correspondiente a las "Orientaciones y Programas para Parvulario y Ciclo Inicialm editadas por la Dirección General de Enseñanza Primaria de la Generalitat de Cataluña ${ }^{3}$.

\section{RESULTADOS DEL ANALISIS DOCUMENTAL}

Hemos de precisar desde un principio que nuestra única finali. dad al analizar el documento citado es estudiar en qué niveles ha in. cidido la obra de Piaget, los aspectos que se retienen de su teoría y la concepción de la relación existente entre el saber de las diferentes disciplinas psicológicas y el saber específico de la psicología educativa, implícita en la forma de utilizar los resultados de la psicología genética en un contexto educativo.

Estudiaremos en primer lugar las repercusiones posibles a nivel de orientaciones psicológicas $y$, a continuación, nos ocuparemos de las influencias que puedan haber en los programas de las diferentes áreas.

A nivel de las orientaciones psicopedagógicas hay, según decla. raciones de la persona representante del Gabinete de Ordenación Educativa (G.O.E.) que entrevistamos, un interés en que éstas abarquen todo el subperiodo que Piaget llama ide las representaciones preoperativas). Por ello, en lugar de ceñirse a dar unas orientaciones acerca de la educación del niño de 5 a 8 años, las plantean como un bloque que comprende desde los 3 a los 8 años. De esta forma, se evita un corte en la exposición de las características evolutivas del sujeto de la educación de Parvulario y Ciclo Inicial. Las orientaciones están estructuradas en los siguientes apartados: 
1. La individualización y la socialización: un proceso esencia.l

1.1 Educación social y cívica.

1.2 Comunicación.

1.3 Actividad lúdica.

1.4 Expresión y educación artística.

1.5 Educación para la salud.

1.6 Iniciación a los conocimientos culturales.

2. Atención psicopedagógica

3. Evaluación.

4. Relación de la escuela con la familia y el entorno.

5. Papel del maestro.

6. Conclusión.

A lo largo de la lectura de estos puntos no aparece ninguna referencia explícita de las ideas de Piaget. La persona entrevistada afirmó, sin embargo, que todo el mundo tuvo en cierta medida presente a Piaget en el momento de confeccionar las orientaciones y programas, aunque no hubiese habido una lectura específica del autor para ver qué se aplicaba. Vamos a intentar detectar algunas de estas influencias implícitas.

En el apartado referente a «La individualización y la socialización: un proceso esencial), se afirma que de los 3 a los 5 años (ces importante que la escuela se plantee la educación de los fundamentos del pensamiento del niño, partiendo del trabajo, de la motricidad, la lengua y la lógica como elementos básicos en la maduración) (p. 58). El cultivo de estos aspectos es lo que le hará capaz al niño de apropiarse de los conocimientos básicos de la cultura que le rodea (pp. 64-65). Se destaca en este sentido la trascendencia de la iniciación en técnicas instrumentales como:

- el conocimiento y dominio del propio cuerpo y de las percepciones espaciales y temporales

- la capacidad de expresión y comunicación

- la capacidad de observación y experimentación

- la capacidad de representación mental y simbólica y las rela. ciones lógicas.

Esto revela, por lo tanto, una intención de influir en el desarro. llo del niño a través de una enseñanza que cultive los aspectos psicomotores, lingüísticos, y lógicos. La finalidad de todó ello es un inte. rés compensatorio y preventivo. Se intenta que el niño llegue a enfrentarse a los aprendizajes posteriores con los requisitos necesarios. Sería en la propuesta de la educación de la lógica donde entre- 
vemos una posible influencia de las ideas piagetianas. Creemos que esta suposición se confirma con el análisis del programa de matemá. ticas que exponemos más adelante.

Respecto a la conceptualización de la actividad lúdica, no vemos en ella una integración de las ideas de Piaget. Consideramos que se está cerca de una concepción funcional:

- se afirma el juego posibilita que el niño se diverta, se evada, reviva situaciones de forma ficticia, se adapte progresiva. mente al grupo...

- se ve en él un medio para conocer las necesidades del niño; así, a partir de la observación del juego, el educador cobtiene información sobre cómo es el niño, cuál es su comportamiento, sus ilusiones y reacciones), y ello permitirá al maestro ciniciar o plantear aprendizajes a partir de lo que los niños hacen, y no tan sólo de lo que los adultos creen que han de hacer o saber) (p. 61)

- igualmente, se le considera como algo más que una descarga de energía superflua: (c...en là escuela el juego no puede que. dar limitado a los ratos pasados en el patio, que sería preciso considerar como tiempo de recreo al aire libren (p. 61). En este sentido, se sostiene que la actividad escolar "se ha de centrar mucho en el juego y proporcionar, siempre que sean necesarios, pequeños tiempos libres, a fin de que el niño pueda seguir un ritmo adecuado (p. 61).

A partir de estas orientaciones, las actividades del niño en el aula quedarían planificadas en los tres bloques siguientes: a) trabajo (tareas en las que se adquieren los objetivos propios del ciclo); b) ac. tividad libre (momentos en los que el niño organiza y estructura su tiempo); y c) patio o recreo (juegos fuera del aula). Esta división es la que Forns (1981, Cap. 2) identifica como características del movimiento de la escuela activa. Dentro de esta corriente, la actividad libre equivale a la actividad funcional, es decir, a la acción que tiene como causa la necesidad del niño y tiene como finalidad su satisfacción. Sin embargo, al no darse todavía una conceptualización ade. cuada de esta actividad (no se le atribuye un papel estructurante en la adquisición de conocimientos) se le atribuyen únicamente la finalidad de movilizar los intereses del niño, adaptarse a la escuela, tranquilizar al niño... y hace falta que haya trabajos impuestos por el profesor para que se dé el aprendizaje. Aquí, en las orientaciones, sólo se habla de (pequeños tiempos libres) que tienen por finalidad que cada niño termine a su propio ritmo el trabajo. No se concibe la actividad libre del niño como elemento a partir del cual se organice 
la jornada escolar y como marco en el que se inserten las intervenciones del profesor que ayuden al niño a organizar la realidad y descubrir las relaciones existentes entre las cosas. No hay, por lo tanto, ninguna relación entre esta actividad lúdica con la actividad autoestructurante del alumno, concebida como fuente de adquisición de conocimientos y que está en la base de algunas propuestas pedagógicas piagetianas (Coll, C. 1981).

A continuación de las orientaciones psicopedagógicas, se propone la globalización de la enseñanza como medio para desarrollar aquéllas. Vemos una posible influencia de la teoría genética en la distinción que se hace entre la globalización entendida como punto de partida y la globalización como objetivo. Dentro de la primera forma de entender la globalización, la estrategia básica es partir de un todo e irlo analizando por medio de las diferentes disciplinas (música, matemáticas, plástica, ciencias...). Este tipo de globalización no se considera adecuado, a pesar de su actual vigencia entre los maestros, debido a que (el esfuerzo de abstración es pobre y propor. ciona más bien un conocimiento de conexión). (p. 77).

Con el término de globalización como objetivo, que es el que se estima como más pertinente, se hace referencia a que el niño pequeño percibe los objetos de una forma global de conjunto. Se afirma que la primera relación del niño con los objetos uno es de conceptualización, sino de actitud, de conducta, de manipulación, y esto le ofrece una percepción global de los objetos». (p. 77). La escuela será la encargada de hacer que el niño realice cuna segunda operación de análisis o conceptualización, que descompone las cosas, los objetos de estudio, en una sèrie de características, de prioridades, para volver al objeto inicial más enriquecido, completo y profundon. (Id.)

En una publicación posterior del G.O.E. ${ }^{4}$ en la que se pretende dar elementos de reflexión y debate a los enseñantes sobre el tema de la globalización, se explicita la influencia de Piaget en esta segunda forma de concebir la enseñanza globalizada. En la fundamentación psicológica de este tipo de enseñanza se citan a Decroly, Wallon y Piaget. De este último se recogen las siguientes ideas:

«Piaget dice que los niños reconocen los objetos en conjunto, sin analizar ni captar los detalles. En esta edad el niño no tiene la suficiente madurez para expresar la realidad global que ha captado. Así pues, a la hora de expresar. se, sólo enumerará, aunque lo haya captado globalmente. Presenta dividido aquello que interiormente tiene unido de una manera perceptiva. Aún no capta la realidad de una manera coherente como un todo. Más adelante, cuando entienda que la realidad está estructurada y sea capaz de explicarla como tal, estará ya en un estadio superior de inteligencia). (p. 20).

A nuestro juicio, es cierto que una propuesta inspirada en Pia. get propondrá una enseñanza globalizada; ahora bien, en lo que no 
estamos de acuerdo es en los aspectos de la teoría genética que se recogen para fundamentar una enseñanza de este tipo. Nos parece entender que la cita anterior se basa en una mala interpretación del pensamiento sincrético del niño preoperatorio. Lo característico de este pénsamiento es que, o bien el sujeto capta lo real de una forma muy global, perdiendo de vista los detalles, o bien capta algún detalle particular y olvida la vision de conjunto. Según nuestra opinión, la razón de propugnar una enseñanza globalizada no reside en que ésta debe adaptarșe a la manera que tiene el alumno de acercarse al conocimiento de la realidad. El alumno capta lo real asimilándolo a sus estructuras de conocimiento. Es precisamente esta referencia a un todo organizado lo que confiere este aspecto global a nuestro conocimiento.

En cuanto a los programas de las diferentes áreas, es en el de Matemáticas donde advertimos una influencia de las ideas piagetianas. A pesar de que se nombra a Piaget sólo en el apartado de geometría, al hacer referencia a las nociones topológicas (p. 214), creemos que su influencia se hace patente también en el resto de los apartados. Analizaremos, en primer lugar, las repercusiones a nivel de contenidos de los diferentes apartados y, en segundo lugar, aquéllas que aparecen a nivel de las orientaciones didácticas que encabezan cada apartado.

Los objetivos básicos de Matemáticas aparecen divididos en cuatro apartados: Lógica, Cálculo, Medida y Geometría. En el apartado de Lógica de Parvulario, aparecen, entre otros, los objetivos de contenido siguientes, que consideramos relacionados con las nociones estudiadas por la psicología genética:

- La actividad clasificadora (que será la base de la formación de los conceptos). (p. 201).

- La actividad ordenadora que cestá en la base de la organización del pensamiento matemático y prepara también en par. ticular la actividad de contarı (p. 202).

Y en el mismo apartado de Lógica, pero refiriéndose al Ciclo Inicial:

- Noción de inclusión y conjunto complementario.

En el ápartado de Cálculo de Parvulario encontramos:

- Favorecer el nacimiento de la idea de número natural. Se propone para este fin practicar el esquema de corresponden. cia como base para la comparación del número de elemen. tos de dos conjuntos. 
En el apartado de Medida para el nivel de párvulos se incluye:

- Hacer expēriencias con materiales continuos: agua, arena (p. 211)

Y, finalmente, entre los objetos de contenido de Geometría para Parvulario, encontramos (pp. 215.216) las siguientes nociones topológicas:

- Aprendizaje del término abierto y cerrado, en un contexto familiar

- Trabajar las nociones (dentro) y (fuera»

y para Ciclo Inicial:

- adquirir la noción intuitiva de frontera y región en la línea, en la superficie y en el espacio (p. 217).

A nivel de orientaciones didácticas referentes a cómo llevar adelante este programa de Matemáticas, encontramos junto a prescripciones que no puede afirmarse en principio que hayan sido extraídas de la teoría de Piaget, como son las siguientes:

- los ejercicios se han de hacer con material tangible, evitando la fijación en las fichas

- fomentar el descubrimiento

- que las nociones no se estudien en sí mismas, sino como ins. trumentos para el juego matemático

otras orientaciones que sí consideramos relacionadas con dicha teoría:

- insistencia en practicar la reversibilidad:

«Es importante en todos los ejercicios reversibles que el niño practique esta reversibilidad. Asi, por ejemplo, cuando ordenemos de grande a pequeño, ordenemos después de pequeño a grande; cuan. do formemos conjuntos con un atributo, hagamos después el ejercicio de encontrar el atributo que ha servido para formar un conjun. tom. (p. 201)

- se caracteriza al niño de Parvulario como no poseyendo la invariabilidad del número de elementos de un conjunto, cuando se cambian de posición. Se recomienda por ello que los cuente una y otra vez (p. 205).

- igualmente se afirma que en Parvulario cla no conservación de la cantidad continua, que se resolverá del todo al final del $\mathrm{Ci}$. clo Inicial, es un obstáculo real que imposibilita la noción'de medida de una cantidad continuaus (p. 211) 
- dentro también dél apartado de Medida (Parvulario), se dice que cla no diferenciación inicial de volumen y peso hace que las comparaciones de pesos no den resultados claros, si la evidencia de una gran diferencia de volumen es contraria (com. paración de objetos de diferente densidad $)$ (p. 211)

- finalmente, en el programa de Geometría de Parvulario, ha. llamos la siguiente cita en las orientaciones didáctičas:

"Cuando hablamos de topología en parvulario, nos referimos al sentido que da Piaget a esta palabra, como término más apto que encuentra para poder describir las primeras nociones de espacio que tiene el niño.

Por propiedades topológicas podemos entender aquellas que se conservan en las deformaciones elásticas) (pp. 214.215).

$y$, a continuación, se afirma que el niño, desde los 3 a los 5 años:

(va incorporando progresivamente a su (esquema) mental del espacio nociones como las de abierto y cerrado, dentro y fuera, antes de adquirir la noción de forma como elemento invariante de las transformaciones rígidas...) ( $p$. 215).

En los programas de las restantes áreas no encontramos una influencia tan clara de la obra piagetiana.

\section{DISCUSION}

En el documento analizado hemos hallado que las referencias a la psicología genética son utilizadas como medio para adecuar los contenidos de los programas a las particularidades evolutivas de los alumnos. Cuando encontramos una alusión a la actividad del alum. no como instrumento metodológico, no la vemos relacionada con la actividad autoestructurante que está en la base de algunas propues. tas piagetianas, sino que más bien dicha actividad toma un sentido cercano a la psicología funcional de Claparède.

A la percepción mencionada por adaptar los contenidos escolares a los estadios del desarrrollo, se añade una selección de estos contenidos a partir de las aportaciones de la psicología genética. Hemos visto que esto se hace más patente en los programas de Mate. máticas. Se incluyen como objetivos de contenido las nociones piagetianas de clasificación, inclusión, seración, número natural, nociones topológicas... La finalidad de todo ello es estimular el desarrollo de las estructuras de base para sentar unas bases necesarias para aprendizajes posteriores.

Este grupo de resultados creemos que es una manifestación de la centración en la naturaleza estructural de la teoría genética, as. pecto que la diferencia de las teorías empiristas del desarrollo cognitivo. Estas últimas, debido al paradigma mecanicista que está en su 
base, conciben el desarrollo como algo puramente descriptivo. (Coll, C. 1979, p. 64). El desarrollo no es susceptible de explicar los cambio comportamentales. Estos son debidos simplemente a un proceso de aprendizaje, programable desde el exterior. La consecuencia de todo esto es que las teorías empirístas reducen su contribución a la educación a una simple (tecnología), es decir, se limitan a suministrar técnicas, para fomentar las conductas deseables y eliminar aquellas que son indeseables. Pero no suministran ningún fundamento para calificar una conducta determinada como deseable o in. deseable. En definitiva, pues, los psicólogos educacionales inspirados en una teoría empirista restringen su campo de trabajo a elaborar unas técnicas que faciliten el logro de unos objetivos educativos defi. nidos por otras personas.

Ahora bien, esto no ocurre en el caso de la teoría piagetiana, que responde más bien a un paradigma organicista. Aquí se supone que el ser humano posee una estructura particular que integra sus di. ferentes atributos. El desarrollo se concibe como los cambios cualita. tivos que sufre esta estructura a lo largo del tiempo. La característica importante a retener para integrarla-a nuestro discurso es precisamente que los cambios que sufren las estructuras del individuo "son en cierto sentido unidereccionales, irreversibles y poseen una vección orientada hacia determinados estados finales) (Coll, C. 1979 , p. 65). Es precisamente este aspecto teleonómico el que explica la naturaleza de los cambios evolutivos que se dan en el comporta. miento humano. Sin embargo, el hecho de postular direcciones universales o puntos finales de desarrollo, hace posible la tentación de adoptarlos como fines educativos. En este error incurren las aplicaciones pedagógicas que extrapolan los aspectos estructurales de la teoría piagetiana a la educación. Sin embargo, consideramos que existen al menos tres razones que demuestran la no pertinencia de este interés por estimular el desarrollo a través de la enseñanza de las nociones piagetianas:

1) la misma teoría genética demuestra que este aspecto no debe preocupar a los educadores porque los niños adquieren las estructuras como fruto de su desarrollo espontáneo, a través de sus experiencias cotidianas. El niño desarrolla sus nociones operatorias, y supera los distintos estadios de desarrollo, como consecuencia de su actividad natural de en. contrar una explicación a cada una de las situaciones con las que se enfrenta día a día, dentro o fuera de la escuela. No hace falta proponerle actividades especiales que estén relacionadas con cualquiera de las nociones estudiadas por Piaget, el niño desarrollará sus marcos básicos de una forma tan natural como aprender la marcha. (Duckworth, E. 1979, p. 304). 
2) aún suponiendo que los niños de los primeros cursos adquiriesen las competencias intelectuales típicas del pensamien. to operatorio concreto, quedaría por determinar qué facto. - res influyen en que esa competencia se actualice o no una si. tuación determinada. El hecho de que un grupo de alumnos haya alcanzado el mismo nivel de desarrollo operatorio no implica que todos utilicen sus marcos de referencia de la misma forma. La cuestión pertinente es saber qué factores influyen en lo que los alumnos hacen con sus estructuras. Esto nos hace pensar que

3) el hecho de haber adquirido las nociones operatorias no hace que el niño comprenda y resuelva automáticamente los problemas implicados en los aprendizajes particulares. En el caso del aprendizaje de las matemáticas son necesarias todavía investigaciones precisas que expliciten la relación psicológica que existe ente las estructuras espontáneas del niño (agrupaciones lógicas de clases, agrupaciones de relaciones, nociones topológicas) con las estructuras que están en la base de la matemática moderna (estructuras algebraicas, estructuras de orden, estructuras topológicas).

Además, se nos hace presentes dos dificultades más con las que se topa un intento de constituir las nociones operatorias en objetivos educativos. En primer lugar, a esta tentativa de inducir a los alumnos a un tipo de pensamiento concreto (o formal) le hace falta una descripción de ejemplos de estrategias de razonamiento concreto o formal tal como se pueden dar en el curso de la actividad de los estudiantes. Sin esto, el educador no puede comprender la naturaleza específica de las competencias que se intentan provocar y por qué determinadas actividades son capaces de provocarlas. En segundo lugar, y lo que es más importante, la elección de los objetivos educa. tivos y la decisión del nivel de profundidad aconsejable deben ser hechas en relación a las finalidades educativas y no sólo contem. plando los avances de la teoría genética, sus campos de estudio o sus conclusiones... La lengua, la historia o la literatura son productos sociales y el contenido de estas ramas del saber no es derivable de la teoría genética. Incluso los objetivos específicos de una disciplina más cercana al campo de estudios de la psicología genética, como puedan ser las matemáticas, deben ser elegidos a partir de una reflexión acerca de qué elementos de esta rama del saber se utilizan en las actividades de los miembros de la sociedad en la que se inserta el proceso educativo.

Todo lo dicho hasta el momento no quiere decir que la consideración de los aspectos estructurales de la teoría para adecuar los contenidos a las características evolutivas del niño no. haya tenido sus efectos positivos. Por ejemplo, en el caso de las Orientaciones y 
Programas de la Generalitat, que están elaboradas en base a las características del subperiodo piagetiano de las representaciones preoperatorias (3.8 años), se há conseguido unificar la programación de Parvulario y Ciclo Inicial y, además, se asegura una iniciación progresiva a los conocimientos culturales, inentando suministrar a los niños técnicas instrumentales imprescindibles sobre la base de un apoyo del correcto desarrollo de las posibilidades personales. Esto asigna a este periodo una función preparatoria, preventiva, y com. pensatoria muy esencial, pues, al asegurar unos objetivos básicos de referencia que deben estar asumidos a fines del ciclo inicial, queda abierta la posibilidad de compensar las diferencias individuales pro. venientes de las desigualdades sociales y culturales.

Sin embargo, esta forma de utilización de la teoría genética se queda a nivel de organización general de la estrategia educativa y no considera la posibilidad de aprovechar las aportaciones de aqué. lla para orientar la gestión de otros momentos del proceso de ense. ñanza-aprendizaje (análisis de tareas y contenidos, descripción del estado inicial del alumno, métodos de enseñanza, sistemas de evaluación). Tener presente este aspecto conduciría a intentar adecuar los métodos de enseñanza a los procesos cognitivos, afectivos y relacionales responsables del aprendizaje, y obligaría a tener en cuenta las investigaciones realizadas, en el marco de la Escuela de Ginebra, desde un paradigma funcional ${ }^{6}$. Situar los resultados de estos trabajos como marco de referencia para el diseño de investigaciones cen. tradas en la resolución de problemas propiamente psicopedagógi. cos, puede ayudar à la psicopedagogía a precisar sus modelos de intervención. Concretamente, consideramos que las aportaciones de estas investigaciones se integrarían dentro de un modelo de orienta: ción cognitivista y èvolutivo que enfatizaría los aspectos de procesamiento de contenidos y los procesos de cambio y desarrollo. Este modelo iluminaría algunos aspectos del proceso de enseñanzaaprendizaje que podríamos esquematizar de la siguiente forma:

1. A nivel de objetivos educativos, se tiene presente que los alumnos ponen en juego diferentes porcedimientos para apropiarse de conocimientos nuevos y que, por tanto, la or. ganización lógica de los contenidos no respeta esa diversidad de estrategias. En consecuencia, se proponen los objetivos heurísticos oformativos como alternativa a los objetivos de contenido. Se trata de establecer y planificar situaciones que permitan a cada alumno ir evolucionando en la construcción de sus propios modelos de adquisición del conocimiento y asimilar también las nociones y conceptos propios de su cultura.

2. A nivel de análisis de tareas y contenidos cobran importancia los trabajos que se están realizando sobre los procedi. 
mientos de apropiación de contenidos específicos (Brun, 1980, 1981 a, 1981 b; Vergnaud, G. y Durand, C. 1976; Vergnaud, G. y Ricco, G. 1976; Vergnaud, G. y otros, 1981). Estos trabajos completarán el análisis lógico de la es. tructura del contenido.

3. A nivel de descripción del estado inicial del alumno, los trabajos que acabamos de citar permitirán conocer, además de los conocimientos que el alumno ya posee sobre el contenido que es objeto de la enseñanza, las representaciones y estrategias subyacentes a esos conocimientos.

4. A nivel de métodos de enseñanza, cobrarán importancia los aspectos referentes al constructivismo y al interaccionismo. Se advierte la necesidad de potenciar situaciones que desencadenen en el alumno actividades cognitivas de tipo estruc. turante.

5. A nivel de evaluación, será posible una nueva concepción de la evaluación formativa (Forns, M., 1980; Allal, L. 1980). Esta consistirá en estudiar los fracasos y éxitos del alumno para conocer el nivel de organización de una determinada noción en que se encuentra el alumno y en intervenir de una forma precisa que consiga movilizar al sujeto hacia la cons. trucción de niveles de organización más elaborados.

Hasta aquí hemos discutido los resultados hallados referentes a la centración en los aspectos estructurales de la teoría genética. Tam. bién hemos hallado, no obstante, una confirmación de nuestra se. gunda suposición inicial concerniente a que encontraríamos una tendencia hacia la extrapolación de los resultados de la teoria genética a la situación educativa. Indicadores de esta tendencia son:

- La caracterización negativa del alumno de parvulario: no posee la invariabilidad del número, no conserva la cantidad continua, no diferencia entre volumen y peso. La razón de esta caracterización negativa radica en que se analizan las conductas del alumno tomando como referencia un modelo terminal (la estructura de agrupamiento que caracteriza el período operatorio concreto).

- El hecho de apoyarse en la caracterización de los diferentes estadios evolutivos para elegir los objetivos y los contenidos (las nociones operatorias) de los programas.

- El proponer actividades didácticas deducidas de las propieda. des de la operación (practicar la reversibilidad siempre que sea posible...) o de las técnicas de investigación (cambiar el agua de un recipiente grande a otro más pequeño, ejercitar el esquema de correspondencia...) 
Para llevar a cabo una discusión crítica de estos datos, es preciso recordar la problemática epistemológica, tan distinta a la educati. va, de la que surgen las aportaciones psicognéticas. Además, el hecho de intentar demostrar la continuidad vida-pensamiento, fuerza a Piaget a estudiar cómo la inteligencia hunde sus raices más profundas en las regulaciones inherentes a todo ser vivo y cómo, a partir de éstas, el individuo va construyendo suś marcos lógicos. matemáticos. Con este planteamiento queda un poco olvidado el estudio de los contenidos de naturaleza social; y la mayoría de los con. tenidos que vehicula el proceso educativo son de este tipo. Estos se adquieren a partir de la interrelación personal y su característica principal es la arbitrariedad, diferenciándose en este sentido del conocimiento físico y del conocimiento lógico-matemático. Asimismo, y como hemos señalado más arriba, los contenidos educativos, su elección y organización, se deducen de los fines y objetivos genera. les de la enseñanza, que tienen una determinación sociopolitica, es decir, surgen del terreno de la ideologia, de la concepción que una colectividad, en un momento determinado de su historia, elabora del mundo, del hombre, de la sociedad.

- De todo lo anterior deducimos, en primer lugar, la no pertinencia de la extrapolación de los datos psicognéticos a la situación èdu. cativa, y, en segundo lugar, que una toma de conciencia de la vocación epistemológica de los datos psicogenéticos, unida a una mayor profundización de la naturaleza de la educación, determinará que las aplicaciones pedagógicas se alejen de la concepción de la extrapolación, y que se emprendan investigaciones propiamente pedagó. gicas, en las que los resultados de la psicología genética sean reela. borados en función de la resolución de cuestiones que intentan hacer más eficaz el proceso educativo. Esto deberá llevar a adoptar una perspectiva situacionista o ecologicista que:

1) estudie las características de la actividad autoestructurante cuando se reviste de los parámetros típicos de una situación educativa concreta,

2) no se ocupe de los instrumentos intelectuales en sí mismos, sino en su utilización para la apropiación de los contenidos escolares,

3) sitúe las aportaciones de Piaget como marco de interpreta. ción de los distintos momentos del proceso de enseñanzaaprendizaje,.

4) y que advierta la necesidad de un complemento de aquellas aportaciones con otras que no estén en contradicción con su interacionismo-constructivismo. 
1 Nos fue de gran utilidad para este fin la comunicación presentada por Josep Puig: «Ciencias de la Educación: diagnóstico y alternativas), en las Jornadas de 'Estudio sobre (Educación, Cibernética y Epistemologían, celebradas los días 29.30 de enero de 1981 en Barcelona y organizadas por el Departamento de Pedagogía Sistemática de la Universidad Central. Las actas de estas Jornadas han sido ya publicadas por el Servicio de Publicaciones de la Universidad de Palma de Mallorca, con el título de aEducación y Cultura, n. ${ }^{\circ}$ ).

2 En un articulo próximo expondremos los resultados principales de los otrös dos apartados correspondientes a las entrevistas y el cuestionario. En nuestra imvestigación tuvimos ocasión de consul. tar otros documentos pero omitimos el resultado de su análisis debido a que todavía no han sido publicados.

3 El texto utilizado por nosotros corresponde a la segunda edición de septiembre de 1981 .

4 Cfr. (Full d'Informació a les Escoles» de 1·12.1981, pp. 19.21.

5 Una breve exposición de lạs principales líneas de investigación en esta línea puede hallarse en el ar. tículo de César Coll y Ch. Gillièron: (JJean Piaget y la Escuela de Ginebra: Itinerario y tendencias ac. tuales" publicado en Infancia y Aprendizaje, Monografía n. ${ }^{\circ}$ : Piaget.

\section{Referencias}

Allal, L.: «Estrategias de evaluación formativa: concepciones psicológicas y modalidades de aplica. ciónn. Infancia y Aprendizaje, 1980, 11, 4.22.

BRUN, J.: «Pedagogía de las matemáticas y psicología: análisis de algunas relaciones»: Infancia y Aprendizaje, 1980,9, 44.45.

BRUN, J.: «Desarrollo cognoscitivo y aprendizaje por objetivos en matemáticas en la escuela primaria». (En COLL, C. [ed.] ): Psicología genética y educación. Barcelona: Oikos-táu, 1981 a, 129.136.

BRUN, J: “La evaluación formativa en una enseñanza diferenciada de las matemáticas». En Coll, C. (ed): Psicología genética y educación, Barcelona: Oikos-tau, 1981 b, 137-146.

Cellerier, G.: El pensamiento de Piaget. Barcelona, Península, 1978.

COLL. C.: «El concepto de desarrollo en psicologia evolutiva: aspectos epistemológicos». Infancia y Aprendizaje, 1979,7,60-74.

COLL, C.: (La psicologia educacional en el marco de las ciencias de la educación», en Coll, C. y Forns, M. (Eds): Areas de intervención de la psicología, Barcelona, Horsori, 1980.

COLL, C.: «Actividad y aprendizaje: aproximación al análisis psicopedagógico de la educación preescolarn. Cuadernos de Pedagogía, 1981, 81-82, 4.7.

COLL, C. Y GILliERON, CH.: "Jean Piaget y la Escuela de Ginebra: itinerario y tendencias actuales.) Infancia y Aprendizaje, 1981, Monografia 2: Piaget, 56.95.

DUCKWORTH, E.: (Either we're too early and they can't learn it or we're too late and they know it already: The dilemma of (Applying Piaget». Harvard Educational Review, 1979, vol 49, 5, 297.312. (Traducción castellana en Infancia y Aprendizaje, 1981, Monografía n. 2: Piaget. 163-176.

Forns, M.: «La evaluación del aprendizaje», en Coll, C. y Forns, M. (eds): Areas de intervención de la psicologia. Barcelona: Horsori, 1980, 105.141.

FORNS, M.: (L'educació pre-escolar, Sentit psicopedagogic de l'activitat lliure». Tis doctoral: Facultat de Filosofía i Ciencies de l'Educació. Secció de Psicologia. Enero 1981.

Generalttat de Catalunya: Orientaciones i programes. Paroulari i Cicle Inicial d'Educació General Básica. Departament d'Ensenyament. Direcció General d'Ensenyament Primari. 2." edició. Setembre 1981.

Generalitat DE Catalunya. G.O.E: «Full d'Informació a les escoles» del 1 de decembre de 1981. 
PUiG, JoSEP M.: "Ciencias de la Educación: diagnóstico y alternativas». Comunicación preséntaida en las Jornadas de estudio sobre "Educación, Cibernética y Epistemologia». Barcelona. 29-30 enero 1981. Educació i Cultura, n. 22, 93-96; Servicio de Publicaciones de la Universidad de Palma de Ma. llorca.

Vergnaud, G. y DuRand, C. "Structures aditives et complexité psychogénetique». Revue Française de Pédagogie, 1976, 36, 28.43. (Trad. cast. en Coll, C.: Psicología genética y aprendizajes escolares. Madrid, Pabla del Rio (èn prensa).

VERGNAUD, G. y RicCo, G.: “Psychögenèse et programme d'enseignement: différents aspects de la notion de hiérarchiem, Bulletin de Psychologie, 1976-77, XXX, 17, 877.882.

Vernaud, G.; HalbWACHS, F. y ROUCHIER, A.: “Estructura de la materia enseñada, historia de las ciencias y desarrollo conceptual del alumnom. En Coll, C.: Psicología genética y educación, Barcelona: Oikos-tau, 1981, 115-128.

\section{Resumen}

Este articulo presenta una parte de los resultados de una investigación realizada acerca de las repercusiones de Piaget en los ambientes educativos. Se presenta el marco general de la investigación y se centra la exposición en el análisis de la influencia en tos diseños curriculares. El texto seleccionado para dicho análisis fue el relativo a las Orientaciones y Programas para Paruulario y Ciclo Inicial editado por la Generalitai de Cataluña.

Los resultadós evidencian una centración en los aspectos estructurales de la teoría genética y una tendencia hacia la extrapolación de los principios y contenidos de la teoria genética a la situación educativa. En la discusión se demuestra cómo una toma de conciencia de la vocación epistemológica de los datos psicogenéticos, unida a una mayor profundización de la naturaleza de la educación. permile concebir nuevos campos de aplicación de la teoría genética, distintos al de la elección y organización de los objetivos educativos, asi como la metodología más pertinente para llevar a cabo esa aplicación.

\section{Summary}

This paper contains a part of the results obtained from a research on the repercussions of Piaget's theory on education. The general frame of the research is presented and the exposition is restricted to the analysis of the influence on one curricula addressed to the preschool level and the early years of the schooling.

The results show that only the structural aspects of the theory are taken into account and that there is a tendency towards the mechanical transposition of the principles and content of the Piaget's theory to education. In the discussion is proved that if the epistemological vocation of genetic psychology is' taken into account and if a profound study of the nature of education is made, it is possible conceive news fields of application of the genetic theory, differents from the objectives of education, and the methodology more suitable for carry out this application.

\section{Résumé}

Cet article présente une partie des résultats d'une recherche réalisée sur les répercussions de la théorie de Piaget sur l'education. On présente le cadre géneral dẹ la recherche et on n'expose que les résultats de l'analyse de l'in. fluence sur les programmes scolaires. Le texte utilisé a cet effet a été celui des orientations et programmes pour l'école maternelle et les deux premiers ans du cycle élémentaire, récemment editées à Catalogne.

Les résultats montrent une centration sur les aspectos strucțuraux de la théorie et une tendence vers l'extrapolation des principes et contenus de celle-lá à l'éducation. Dans la discussion on démontre comment une prise de conscience de la vocation epistémologique des données psychogénetiques et une étude plus profonde de la nature de l'éducation, permettent imaginer de nouveaux domaines d'application de la théorie génétique, différents du choix des objectives éducatifs, et la méthodologie la plus appropiée pour mener à bien cette application. 